\title{
FACTORES MATERNOS Y PERINATALES ASOCIADOS A HIPOACUSIA. SERIE DE CASOS
}

\section{Maternal and perinatal factors associated with hypoacusia. A series of cases.}

Ruth Perez-Villegas, Lic., MSc*, Macarena Arriagada-Belmar, Lic.**, Maribel Aviles-Muñoz, Lic.**, Jazmín Palma-Villegas, Lic.**, Marcela Valenzuela-Maureira, Lic.**

Recibido: marzo 21/06 - Revisado: junio 27/06 - Aceptado: julio 28/06

\section{RESUMEN}

Introducción: la hipoacusia constituye un importante problema de salud pública, debido a su repercusión sobre el desarrollo emocional, cognitivo y social del niño .

Objetivo: determinar factores maternos y perinatales asociados a una población de escolares con hipoacusia.

Población: estudio realizado durante el año 2005 en 79 escolares hipoacúsicos detectados por la Junta Nacional de Auxilio Escolar y Becas (JUNAEB).

Procedimiento: los datos maternos y perinatales fueron obtenidos de las fichas perinatales en el Hospital Herminda Marti de Chillán, VIII Región, Chile.

Variable independiente: hipoacusia

Resultados: el promedio de edad actual de los niños fue 16,5 años y la edad promedio de detección de hipoacusia fue de 6,3 años. El 74,7\% tenía una edad gestacional de término al nacer. El $50,6 \%$ tuvo un peso al nacimiento entre 2.500 3.499 grs. El 38\% presentó algún factor de riesgo

\footnotetext{
* Licenciada en Obstetricia y Puericultura' Especialista en Perinatología y Magíster en Ciencias de la Educación, Departamento Obstetricia y Puericultura. Facultad de Medicina. Universidad de Concepción. Chile. Casilla 160 - C. Concepción, Chile. Fax: 56 - 41 - 204837 Correo electrónico: ruperez@udec.cl

** Licenciada en Obstetricia y Puericultura. Facultad de Medicina. Universidad de Concepción. Chile.
}

perinatal asociado, siendo el principal la hiperbilirrubinemia (22,6\%). La mayoría de los casos de hipoacusia corresponden al tipo bilateral severo (40,5\%).

Palabras clave: pérdida auditiva, trastornos de la audición, factores de riesgo.

\section{SUMMARY}

Introduction: hypoacusia represents an important public health problem, due to its repercussions on emotional, cognitive and social development.

Objective: determining maternal and perinatal factors associated with a student-aged population suffering from hypoacusia.

Materials and methods: a series of cases were studied during 2005; 79 students suffering from hypoacusia were detected by the Junta Nacional de Auxilio Escolar y Becas (JUNAEB) during this period.

Results: the average age of the children being studied was 16.5 years. $74.7 \%$ had gone to full-term gestation on being born. 50.6\% had had 2,500-3,499 g birth weight. $38 \%$ presented some associated perinatal risk factor, mainly being hyper-bilirrubinaemia (22.6\%). The age average for hypoacusia being detected was 6.3 years. Most of the cases corresponded to severe bilateral hypoacusia (40.5\%).

Key words: hearing loss, hearing disorders, risk factor. 


\section{INTRODUCCIÓN}

La hipoacusia constituye un importante problema de salud pública, debido a las repercusiones que tiene sobre el desarrollo emocional, cognitivo y social del niño con su entorno. ${ }^{1-4}$

Los defectos de la audición, congénitos o adquiridos en el período perinatal, constituyen un grave trastorno sensorial que afectará el desarrollo del habla y del lenguaje, ocasionando serias dificultades psicológicas y de comunicación, tanto para el niño como para su familia, lo que lleva a problemas de desarrollo personal y de integración social del paciente. ${ }^{5-8}$

El daño auditivo perinatal es de etiología multicausal ${ }^{9}$ según un estudio realizado por Peñaloza et al (2004), en el que se revisó la casuística total del archivo clínico del Instituto Nacional de la Comunicación Humana (5.732 casos), de estos se seleccionaron 727 casos de hipoacusia-sordera sensorial determinada como congénita o adquirida antes de los cinco años de edad, de ellos, en 160 se observó la asociación del trastorno auditivo con condiciones perinatales adversas, específicamente el peso al nacer, puesto que el $57 \%$ tuvo un peso inferior a 2.900 g. En el 56,3\% (n=90) de los casos estudiados, el parto se interpretó como distócico. Asimismo, en $33,8 \%(n=54)$ de los casos, hubo evidencia de problema neonatales en el niño, como ictericia, cianosis o trastornos respiratorios.

La incidencia de la hipoacusia severa o profunda en el recién nacido, según la OMS es de 1 por mil, ascendiendo al 1-3 por mil si se incluye la variedad moderada y al 5 por mil recién nacidos vivos cuando se consideran todos los grados de hipoacusia., ${ }^{1,5,10}$ En Chile, aun cuando no existen cifras homogéneas acerca de la incidencia de hipoacusia neonatal, ésta oscila entre 1 y 3 casos por cada mil recién nacidos vivos. ${ }^{11}$ Este índice a pesar de no mostrar una alta frecuencia es mayor que las incidencias de otras enfermedades, para las que ya se están aplicando pruebas de detección precoz como es el hipotiroidismo donde la tasa es de 1/3.500 y fenilcetonuria de $1 / 14.000$.
A nivel local, no se encuentran datos que nos permitan conocer la incidencia de hipoacusia infantil en cualquiera de sus grados, los únicos registros existentes son los que mantiene la Junta Nacional de Auxilio Escolar y Becas (JUNAEB), con su programa de salud escolar, entidad que desde 1992 ha tenido dentro de sus principales metas la pesquisa y derivación de los niños con algún tipo de alteración auditiva. Tampoco hay estudios en relación a los factores asociados a la hipoacusia en la infancia.

De esta forma, el objetivo de esta investigación es determinar posibles factores maternos y perinatales asociados a una población con hipoacusia detectada en la etapa escolar.

\section{MATERIALES Y MÉTODOS}

Diseño: serie de casos.

Población y Muestra: de un universo de 177 niños y adolescentes con hipoacusia, pertenecientes al programa de salud escolar, subprograma de hipoacusia, de la Junta Nacional de Auxilio Escolar y Becas (JUNAEB) en Chillán - Chile detectados durante su etapa escolar básica entre los años 1986 -2005, con diagnóstico realizado por el especialista otorrinolaringólogo de la JUNAEB, se seleccionó una muestra secuencial por conveniencia de 79 casos cuyas edades fluctuaron entre 4 hasta 19 años de edad. Para ingresar al estudio debían tener su domicilio en Chillán y haber nacido en el hospital Herminda Marti.

Chillán se encuentra ubicada en la VIII región del Bío-Bío, a 480 kilómetros de la capital de Chile, con una población de 161.953 habitantes, con un $8,7 \%$ que vive en zonas rurales (13.938 habitantes). Debido al clima templado de la zona, la actividad principal es agrícola y vitivinícola. El hospital Herminda Marti, es un centro de salud que cuenta con las especialidades de Medicina, Cirugía, Pediatría y Obstetricia y Ginecología, es un centro de derivación de los hospitales rurales de la provincia de Nuble y tiene un promedio anual de 2500 partos. 


\section{Procedimiento:}

Se recolectaron los datos maternos y perinatales registrados en la historia clínica y de los libros de registros de la unidad de partos, recién nacidos y unidad de neonatología del Hospital. Se diseñó una base de datos utilizando el software Microsoft Access ${ }^{\circledR} 2002$.

\section{Variables estudiadas:}

Hipoacusia: pérdida total o parcial de la capacidad auditiva por uno o los dos oídos. La intensidad del sonido se mide en decibeles (dB) y mide la amplitud del sonido.

Audición normal: audiometría tonal en umbrales entre 1 y 20 decibeles, el rango normal de audición del ser humano es de aproximadamente 20 a 20.000 $\mathrm{Hz}$.

Dada la variación entre las diversas clasificaciones de hipoacusia, se aplicó el criterio utilizado en el protocolo del programa de Hipoacusia del Prematuro, del Ministerio de Salud de Chile, obtenida de la Sociedad Británica de Audiología y Asociación Británica de profesores de sordos 1988, que la clasifica de la siguiente manera: Hipoacusia leve, el promedio de umbrales se encuentra entre 20 y 40 decibeles; Hipoacusia moderada entre 41 y 70.; Hipoacusia severa entre 71 y 95 e Hipoacusia profunda (sordera) con umbrales sobre 95 decibeles. Se clasificó también su localización: localización unilateral o bilateral, el origen: sordera congénita o adquirida. Hipoacusia mixta y sin clasificar.

Como variables pronósticas se analizaron los factores perinatales como edad gestacional al nacer menor a 32 semanas, peso de nacimiento menor a 1500 grs., Asfixia neonatal definida como aquel recién nacido con test de Apgar al minuto menor a 7 puntos; malformaciones y patologías como hiperbilirrubinemia neonatal $(>15 \mathrm{mg} / \mathrm{dl})$, meningitis neonatal definida como cuadro séptico de origen bacteriana diagnosticada mediante estudio del LCR. Tratamientos efectuados como fototerapia y ventilación mecánica. Otras variables consideradas fueron la edad de detección de la hipoacusia o sordera y los antecedentes maternos como edad, paridad, tipo de parto; vaginal o cesárea, causas de cesárea y antecedentes familiares de hipoacusia.

\section{Análisis:}

Para el análisis descriptivo del estudio se utilizaron medidas habituales de tendencia central y de dispersión.

\section{RESULTADOS}

En el grupo estudiado, el promedio de edad al momento del estudio fue de 16,5 años, el 51,9\% de los casos se concentró en el rango de 15 a 19 años, el 53,2\% pertenecían al sexo femenino (42 casos) y $46,8 \%$ al sexo masculino (37 casos).

Con respecto a la edad gestacional al nacer, el promedio de edad gestacional fue de 37,8 semanas, con un rango entre 31 a 41 semanas de gestación. De los 79 niños, 14 nacieron con menos de 37 semanas de edad gestacional (18\%)

La distribución de los casos según el tipo de parto fue de 48 nacidos de parto vaginal $(60,8 \%)$ y 31 de parto cesárea (39,2\%), las principales causas de cesárea fueron desproporción céfalopelvica (29\%), cesárea anterior $(16,1 \%)$ y distocia de presentación (6,5\%). Con relación al Apgar al minuto de vida, la mayoría de los casos $(92,4 \%)$ se concentró en el rango de normalidad ( $>7$ puntos) y un 7,6\% correspondió a asfixia moderada, no se presentó ningún caso de asfixia severa en el total de casos estudiados. El peso promedio al nacer fue de 3.036 g, con un rango entre 720 a 5.020 g. Tres niños nacieron con peso menor a $1.500 \mathrm{~g}(3,8 \%)$ y 12 presentaron pesos menores a $2.500 \mathrm{~g}(15,2 \%)$

Con respecto a los antecedentes maternos, el promedio de edad de las madres fue de 26,7 años, con un rango entre 15 y 43 años, el 21,5\% de las madres eran adolescentes, el 45,7\% era primípara y el 19\% multípara de uno parto anterior.

Del total de los casos estudiados, el 38,0\% presentó al menos un factor de riesgo perinatal asociado al desarrollo de hipoacusia, siendo los más 
Tabla 1. Factores de riesgo en la población con hipoacusia.

\begin{tabular}{|l|c|c|}
\hline Factores de riesgo perinatales & $\mathbf{n}$ & $\%$ \\
\hline Hiperbilirrubinemia & 14 & 22,6 \\
\hline Fototerapia & 12 & 19,4 \\
\hline Ventilación mecánica & 7 & 11,3 \\
\hline Peso < 1.500 grs. & 6 & 9,8 \\
\hline Asfixia neonatal & 6 & 9,8 \\
\hline Malformación craneofacial & 5 & 8,1 \\
\hline Antecedentes de hipoacusia & 3 & 4,8 \\
\hline Meningitis bacteriana & 3 & 4,8 \\
\hline Edad gestacional $<32$ semanas & 2 & 3,2 \\
\hline Rubéola & 2 & 3,2 \\
\hline Toxoplasmosis neonatal & 1 & 1,5 \\
\hline Epilepsia & 1 & 1,5 \\
\hline
\end{tabular}

frecuentes la presencia de hiperbilirrubinemia y los tratamientos en fototerapia y ventilación mecánica (tabla 1). Sólo tres casos presentaron antecedentes familiares de hipoacusia $(3,8 \%)$.

Respecto a la edad de detección de hipoacusia, el 43\% de los casos se detectaron en el rango de 4 a 7 años, un 29,1\% entre 0 y 3 años. Un $16,5 \%$ de los casos la hipoacusia se detectó en la etapa de la adolescencia, entre los 12 y 19 años de edad, el promedio de edad de detección fue de 6,3 años. El 100\% de los casos de sordera congénita ( 3 casos), se presentó en niños que habían nacido con menos de 37 semanas de gestación y con un peso al nacer entre 1.500 y $2.499 \mathrm{~g}$.
De acuerdo a la clasificación de hipoacusia, la bilateral severa se presentó en el 40,5\% de los casos estudiados. De la totalidad de casos detectados entre los 0 y 7 años de edad, el 50,9\% (57 casos) fueron del tipo bilateral severa (tabla 2). La hipoacusia del tipo bilateral severa se presentó en niños de término en un 81,2\%.

\section{DISCUSIÓN}

El presente estudio muestra una alta frecuencia de sordera severa bilateral y la presencia de múltiples factores de riesgo perinatales en los niños con hipacusia en edad escolar. Concuerda con la literatura actual, donde se señala que el daño auditivo no es unicausal, la interacción entre factores de riesgo es común cuando ellos están presentes, encontrándose en algunos casos más de un factor de riesgo. ${ }^{1}$

El desarrollo neural de la vía auditiva central se ve afectado por múltiples factores, sin embargo, de acuerdo a varios estudios, desde un 30 a un 77,4\% de los casos no se encuentra una etiología que justifique su alteración, situación que podría explicar en parte nuestros hallazgos, puesto que un $62 \%$ de los casos estudiados no presentó factores de riesgo y los que se presentaron, no eran específicos para el desarrollo de hipoacusia.

La presencia de hipoacusia-sordera puede ser el resultado de situaciones que han estado

Tabla 2. Edad de detección y tipo de la Hipoacusia.

\begin{tabular}{|l|c|c|c|c|c|c|}
\hline \multicolumn{1}{|c|}{ Edad de detección (años) } & \multicolumn{2}{|c|}{$\leq \mathbf{7}$} & \multicolumn{2}{c|}{$\mathbf{8 - 1 9}$} & \multicolumn{3}{c|}{ Total } \\
\hline Tipos de Hipoacusia & $\mathbf{n}$ & $\mathbf{9}$ & $\mathbf{n}$ & $\mathbf{\%}$ & $\mathbf{n}$ & $\%$ \\
\hline Unilateral leve & 1 & 1,8 & 0 & 0 & 1 & 1,3 \\
\hline Unilateral moderada & 1 & 1,8 & 6 & 27,3 & 7 & 8,7 \\
\hline Unilateral severa & 2 & 3,5 & 1 & 4,5 & 3 & 3,8 \\
\hline Bilateral leve & 4 & 7 & 0 & 0 & 4 & 5,2 \\
\hline Bilateral moderada & 10 & 17,5 & 6 & 27,3 & 16 & 20,2 \\
\hline Bilateral severa & 29 & 50,9 & 3 & 13,7 & 32 & 40,5 \\
\hline Bilateral mixta & 2 & 3,5 & 1 & 4,5 & 3 & 3,8 \\
\hline Sordera congénita & 3 & 5,2 & 0 & 0 & 3 & 3,8 \\
\hline Sin clasificar & 5 & 8,8 & 5 & 22,7 & 10 & 12,7 \\
\hline Total & $\mathbf{5 7}$ & $\mathbf{1 0 0}$ & $\mathbf{2 2}$ & $\mathbf{1 0 0}$ & $\mathbf{7 9}$ & $\mathbf{1 0 0}$ \\
\hline
\end{tabular}


presentes en la madre desde el momento de la concepción, antes del parto, durante la atención de éste o en el periodo neonatal. ${ }^{6}$

Entre los factores de riesgo identificados, el peso de nacimiento menor de $1.500 \mathrm{~g},{ }^{4,6,9,12}$ ya sea bajo peso para la edad gestacional o recién nacido prematuro adecuado para su edad gestacional. Aunque también se ha relacionado con pesos más elevados al nacer. ${ }^{5,6,13,14}$

Otros factores de riesgo son la hiperbilirrubinemia, ${ }^{4,9}$ la historia familiar de hipoacusia hereditaria, desarrollada antes de los 50 años de edad en familiares directos y sin causa aparente, las enfermedades infecciosas como TORCH, ${ }^{4,5,10,15}$ la hemorragia intracoclear e intracraneal se relacionan a menudo con hipoxia perinatal. ${ }^{16}$

La frecuencia de factores de riesgo positivos en nuestro reporte es del 38\%, lo que coincide con algunos trabajos previos, pero es inferior a otras publicaciones. ${ }^{11,17,18}$ Este resultado puede verse influido por la poca importancia que se da a la búsqueda de factores de riesgo y su consignación en las historias clínicas y perinatales, pues algunos de ellos, especialmente los antecedentes familiares de hipoacusia y/o sordera, no son registrados de forma específica.

Se considera que la manifestación más común de hipoacusia secundaria a factores adversos perinatales es la de tipo severa, usualmente bilateral, que compromete el aprendizaje del lenguaje, requiere de terapia con especialistas y de la utilización de auxiliares auditivos eléctricos. ${ }^{9}$ En nuestro estudio, el 40,5\% de los casos presentaron hipoacusia bilateral severa.

Sin la realización de una tamización universal para la detección precoz de la hipoacusia, la edad media del diagnóstico se sitúa alrededor de los tres años, cuando los padres o educadores comienzan a detectar las primeras manifestaciones. En nuestros resultados, la edad de mayor pesquisa corresponde al rango entre 4 y 7 años, lo que demuestra la tardanza en su detección y por lo tanto en su manejo, ${ }^{6}$ queda planteada la necesidad de realizar un cribado que incluya a todos los recién nacidos, antes de que se desarrollen trastornos en la adquisición del lenguaje.

\section{REFERENCIAS}

1. Gallardo M, Vera C. Estudio de la vía auditiva central por medio de las respuestas evocadas auditivas del tronco encefálico (ABR), en niños con retraso en el lenguaje. An Fac Med 2003;64:27-33

2. De la Paz V, Miranda M, Rojas L. Consideraciones audiológicas. En: iAlumno sordo integrado! Guía para profesores de educación general. Santiago, Chile: Editorial universitaria; 1993. p. 17-23.

3. Arruti I, Pélach R, Zubicaray J. Hipoacusias en la edad infantil. Diagnóstico y tratamiento. Anales Sis San Navarra 2002;25 (supl. 2):73-84.

4. Ministerio de Salud. Garantías explicitas en salud. Hipoacusia Neurosensorial bilateral del prematuro. Guía clínica. Chile: Publicación oficial; 2005.

5. Rivera T. Detección precoz de la sordera. ORL-DIPS 2003;30:128-32.

6. Peñaloza Y, Castillo G, García F, Sánchez H. Hipoacusiasordera asociada a condiciones perinatales adversas según registro en unidad especializada de la ciudad de México. Análisis en función del peso al nacimiento. Acta Otorrinolaringol Esp 2004;55:252-9.

7. Delprat B, Ruel J, Guitton MJ, Hamard G, Lenoir M, Pujol R, et al. Deafness and cochlear fibrocyte alterations in mice deficient for the inner ear protein otospiralin. Mol Cell Biol 2005;25:847-53.

8. Sano M, Kaga K, Kitazumi E, Kodama K. Sensorineural hearing loss in patients with cerebral palsy after asphyxia and hyperbilirubinemia. Int J Pediatr Otorhinolaryngol 2005;69:1211-7.

9. Martínez CF, Poblano A, Fernández LA, Garza S. Factores de riesgo para hipoacusia y hallazgos audiométricos en una población preescolar egresada de cuidados intensivos neonatales. Salud Publica Mex 1995;37:205-10.

10. Ferrer E, Tobón G, Guerra L. Evaluación de la hipoacusia neurosensorial en el neonato. Acta de Otorrinolaringología \& Cirugía de Cabeza y Cuello 2004;32:127-30.

11. Cabra J, Moñux A, Grijalba M, Echarri R, Ruiz E. Implantación de un programa para la detección precoz de hipoacusia neonatal. Acta Otorrinolaringol Esp 2001;52:668-73. 
12. Aránguez G. Diagnostico de la hipoacusia infantil. An Pediatr (Barc) 2003;Monog 1:24-8.

13. Lichtig I, Monteiro SR, Couto MI, Haro FM, Campos MS, Vaz FA, et al. Assessment of auditory behaviour and neuropsychomotor development of low weight infants. Rev Assoc Med Bras 2001;47:52-8.

14. Van Naarden K, Decoufle P. Relative and attributable risk for moderate to profound bilateral sensorineural hearing impairment associated with lower birth weight in children 3 to 10 years old. Pediatrics 1999;104:905-10.

15. Menéndez I, Ponce M, Carrillo B, Gil J. Sorderas neurosensoriales no sindromáticas. Análisis de la herencia en 10 familias. Rev Cubana Pediatr 1998;70:92-9.

16. Carrasco J, García M. Papel de la matrona en la prevención de la sordera congénita secundaria a hipoxia fetal. Enfermería Integral 2001;56:15-7.

17. González A, Bonilla C, Morales C, Gómez F, Barrasa J. Cribado universal de la hipoacusia congénita en Cantabria (España): resultados de los dos primeros años. An Pediatr (Barc) 2005;62:135-40.

18. Martinez R, Benito JL, Condado MA, Morais D, FernandezCalvo JL. Results of the application of the protocol for the early detection of hearing loss in high-risk neonates.An Otorrinolaringol Ibero Am 2003;30:277-87. 